\title{
Innovative Flipped Classroom Strategy by Computational Thinking
}

\author{
Zhen Kong ${ }^{1, ~ a}$, Kun Bai ${ }^{2,}$ b, *, Linghan Kong ${ }^{3}$ \\ ${ }^{1}$ Beijing Information Technology College, No. 5, Fangyuanxilu, Chaoyang District, Beijing, China \\ ${ }^{2}$ University of International Relations, No. 12, Poshangcun, Haidian District, Beijing, China \\ ${ }^{3}$ Beijing Bayi School, No. 29, Suzhoujie, Haidian District, Beijing, China \\ ${ }^{a}$ Kongz@bitc.edu.cn, ${ }^{b}$ Baikun@uir.edu.cn
}

Keywords: Flipped-classroom; pedagogy; innovation; education; computational thinking.

\begin{abstract}
The rapidly increasing use of computers in education has caused a resurgence of interest among educators. Computational thinking is going to be a defining feature of the future-and it's an incredibly important thing to be teaching today. There's always lots of discussion (and concern) about how to teach traditional mathematical thinking to students. But look to the future, this pale in comparison to the importance of teaching computational thinking. But computational thinking is going to be needed everywhere. And doing it well is going to be a key to success in almost all education settings. Educational technology must be engaging, interactive, and motivating. Integrating information and communications technologies into the classroom examines topics critical to innovation and entrepreneurship. The ideal model for flipped instruction stands on four pillars: a platform for instructional delivery, availability of student and faculty resources, creation of an online community, and faculty assessment. An educational technology that incorporates a high degree of social and community-building opportunities is more effective and successful.
\end{abstract}

\section{Introduction}

In recent years, engineering courses have been facing a double challenge; they must deal with the high velocity of technological changes and, at the same time, they must provide engineers with skills that some years ago were acquired from the professional experience, such as the capacity of teamwork, leadership, communication, and expression. These abilities are being called as "transversal competencies” or even "generic competencies”, "transferable competencies", “core competencies", "professional competencies" and always refer to those competencies that are beyond technical subjects but are also related to professional practice. In seeking to explain the significant increase in the use of flipped classroom in colleges, three drivers are proposed. First, the explosion of social software has become so prevalent among teenagers and adults. Second, declining student numbers in colleges coincides with pressure on educators to provide a wider and more varied curriculum. Third, technology can have a significant role to play in broadening choice through the online delivery of distance learning to courses traditionally delivered face-to-face to a single class gathered in one room. The latest concept evolving in pedagogy is flipped classroom utilized for active learning by students with their peers and faculty. It uses class time for engaging 
activities and instructor-guided problem solving. In class, the concepts from the video lectures are applied to problems, and students are challenged to think beyond given examples. Students show increased comprehension of the material and appeared to improve their performance. Students become noticeably passionate and more comfortable with the subject. In addition to being an effective tool for teaching, flipping the classroom may help students take more ownership of their learning. It is possible to develop a fully internally consistent model for an isotropic elastic body that has nonlinear response relations but with small strains.

\section{Computational Thinking as the Skill}

We are entering into the ubiquitous computing age now. Cyberspace community of shared destiny has become a buzzword since the Second World Internet Conference. The phrase voices principles of opening, sharing and transparency of the Internet. Thus, promoting interconnectivity is essential to bridge the information gap between different groups. People communicate through the Internet, leading to another vast data source. Computational thinking is now widely acknowledged as a necessary skill to survive in 21st century. If computational thinking is possible, computational learning is either possible. Computer-based simulation can be used to facilitate learning concepts more thoroughly and effectively. This demands a fundamental change in the conventional lecture-based pedagogy.

\subsection{Eternal Memory by E-learning}

Technology provides firsthand experience rather than reading from a textbook, and therefore becomes a more memorable and exciting learning experience. The aim of teaching is to create knowledge and competence. Maintaining knowledge, avoiding forgetting of prior knowledge, is another aim. By computers, it simulates the cognitive processes of humans in storing information, solving problems, meta cognition, detecting inconsistencies, etc. The human memory and the memory traces are the basis and the substrate of human learning and knowledge. Thus, in addition to psychology and giving immediate feedback after each learning step, e-learning is now popular due to the reinforcement on students' memories. Certainly psychology of memory has the potential to improve e-learning systems. The methods and results for multi-attribute coding, multiple-modality coding and deeper levels of processing are the basis for optimal coding and adequate rehearsal strategies. The different kinds of cognitive representations, e.g. pictorially, verbal, are important for cognitive functioning in learning, problem solving and creative processing. The same content can be represented mentally in different ways. It facilitates the generation and interpretation, as well as the flexible and creative usage of the suitable representations by e-learning for problem solving and storage. Recall and recognition in answer formats is of great importance. According to 3-years study in Beijing Information Technology College, lectures as a teaching method are ineffective and it is not at all an efficient communication method. Learners can simply forget what is told at 10 minutes back and there is no way to rewind it for rehearing. On the other hand, online videos and growth of internet are boosting the implementation of flipped class room. The research team find that over 65 percent reported re-watching the lectures to clear up misunderstandings. The following suggestions for faculty interested in flipping: (1) Keep the video segments less than 10 minutes, (2) review the material in class for less than 20 minutes, (3) give students time in class to work on real-life and relevant problems or projects that are traditionally done at home, and provide at least weekly assessments to keep the students on track. 


\subsection{Web-based System}

Web is changing the educational pedagogy, requiring new ways of assessing, mentoring, and facilitating education. Self-centered learning is becoming the norm. Moving from traditional teaching to the Web-based system requires mapping or transformation from one medium to another. The system should address the many aspects of the teaching and learning processes. It is available anytime, anyplace, to anyone-irrespective of time and distance. Administrators and educators need to provide a seamless online web operation. There are now several tools available for making annotated video lectures and also there are plenty of sources from where one can collect videos for supporting one's own created content. This also facilitates the teacher to concentrate on the weaker section of the class to come up who otherwise do not raise any question. The biggest challenge is making appropriate computational experiments to the subject consideration. Classroom is made as a place to work through problems and for discussion and also for engaging in collaborative learning with peers. A lot of work has been done in designing new methods and tools to support course management and communication between professors and students. Different solutions have been proposed to simplify student access to class material, to help students submit the results of exercises, to help professors distribute lecture notes, to get student feedback, and to monitor student progress. A professor may easily adjust the system to meet the teaching needs. The interface is intuitive. Both instructors and students need not waste time in becoming familiar with the communication technology. We use Java to create a web-based application to implement aforementioned communication functions. The HTTP protocol is used for all communications between users and the TSI, allowing participants to employ standard Web browsers. Scalability, utility, dissemination, and portability are important criteria for exemplary educational system. A technology should be able to handle a large number of students and reach a large audience, but it should also adapt easily to other contexts and across different platforms.

\section{Open Access as the Chance}

Internet-based programs allow students to take notes and access resources online, receiving extra support at home. This gives a chance to expand the school without stressing staff out. It is maximizing the ability to teach. Flipping the classroom allows an educator to record a lesson plan on video in the same fashion it would be presented to students. Students can access the lessons on any computer or smart phone. Other benefits of flipping include: students are less frustrated and disruptive in class because one-on-one help; a much larger percentage of assignments are completed and to a much higher quality. Educators and leaders are transforming lower-performing schools by professional practice, collaboratively changing the strategies to an approach that is modern, relevant, and student centered. Technology must be lower end enough to work within the college's resources. Therefore, the flipped materials can reach a larger audience. For example, the iPod is an exemplary educational technology that has gained success because of pervasive use and ease to the classroom. The open access is an important feature for exemplary technology because it can make a big impact when it is used on a widespread basis.

\subsection{Micro Class as the Innovation}

As Chinese society continues into the internet age, more modern pedagogical approaches to teaching should be explored. Traditional lecture classes may fall short on student engagement, learning and retention of the subject matter. Micro-classes and micro-courses have become a focus and hot spot of the construction of teaching resources. With advancement in technology, this model does not overlook the diversity of student learning. There is growing interest in hybrid, blended, and 
flipped instruction as a way to incorporate the best of different delivery methods. The data further indicates the most positive impact with online learning experiences is the class structure that supports flexibility, organization, and clear expectations. Nontraditional students reported more positively than traditional students about the benefits of flexible micro classes with clear course structures[4]. It is being developed and offered worldwide. Many people throughout the world are interested in equine-related material. When students are asked to evaluate their experiences in the flipped format of the micro class, an overwhelming majority indicate it fosters a better learning environment with a more effective teaching strategy. It is proving to be a teaching approach that students appear to benefit from and embrace [5].

\subsection{Deep Learning as the Focus}

Students can employ deep learning approaches, and educators can establish curricula that promote deeper learning of concepts, which is seen to be preferable in most cases. In a deep learning approach, students learn for understanding by interacting with course content and by relating ideas to previous knowledge and experience, whereas surface learning is largely dependent on memorizing course content without necessarily seeking to understand its logic or meaning. Moreover, materials learned are retained longer. Flipped format can encourage deep learning. Flipped classrooms provide an online format, becoming workshops of learning. The instructor may support the pre-learning process by simply providing recorded lectures or, if deep learning is required or given as an option for students, through the inclusion of interactive exercises and online quizzes. It is a result of a long search for an effective way to shift learning to the hands of the learner. A lot of advantages range from meeting students in their digitally-saturated world, answering needs for timing of core online learning, supporting struggling and advanced students to work at their own pace; and increasing interactions between students and teachers. Students change from the product of one-way information input, to the centre of learning, where they actively participate and engage in class work that can maximize deep learning.

\subsection{Further Learning as the Goal}

Technology has the power to bring people together and to greatly enhance further learning via collaboration. Education technology moves away from text-based to engaging game and simulation education. Engagement is extremely important for getting students interested in further learning. Thanks to videogames and communication tools, technology already is prevalent in many students' lives, and can be highly motivating and conducive to student learning. Likewise, interactive technology that allows students to exchange work, ideas, and data and that also allows for multiple platforms provides exemplary educational experiences. Students who see and analyze real-time data are much more engaged and interested in what they are learning, just because the environment is dynamic. More learning takes place, students remember more, dig deeper, and go further. Students are actually learning more in the situated learning perspective. This type of tool lets students access current data and information and build a social network around their learning task. Communication technologies that enable students to access information and resources from global intelligence pools will quickly revolutionize how students work through academic courses, obtain information, and collect their own data. Students will communicate with mentors and peers in other countries, unconstrained by geography. Social networking technologies will provide invaluable expertise and support as they take advantage of distance human interactions and artificial intelligence. The multiple platforms of communication employ multiple social activity structures, provide opportunities for learners to serve as instructors of their peers[6]. 


\section{Learning Framework}

The building of skills and knowledge value via engagement can also be attained, which may be beneficial to the organization. Flipped classroom promote student participation, engagement and co-creation. Online micro classes improve broad awareness and understanding of the content. This provides opportunities for greater participation. The learning framework entails three core components including an online component, a workshop component and a tutorial component. The online component replaces lectures and includes recorded lectures, lecture slides with notes and audio recordings. It enables students to develop a basic understanding of the content. The face-to-face workshop provides authentic activities, such as case studies, discussion and questions on real world examples. It helps students for decision making. Tutorials are usually groups of 20 approximately students, and become a strong delivery mode for developing and co-creating specific skills value. Enhancement of group collaboration improves students' experiences through the scaffolding of group projects in the class [7].

\subsection{Workshop}

All students independently cover core content that conventionally is covered in a workshop in the same way online. Students participate in workshop activities in groups with expert support and feedback. Meanwhile, students work together in groups and communicate with tutor online in a single session. The presentation skills are developed in this process, which are vital to graduates in the future innovation. This strategy adopts to fulfill the aim of increasing the co-production and co-creation of the targeted graduate attributes and learning outcomes. Workshop activities included: questions relating to short news articles, small case studies, and interactive activities applying concepts, video cases and audits of advertising materials.

\subsection{Tutorial}

There are two versions of an intelligent tutoring system, one is based on an expert model, the other is on a model of an intelligent novice. During problem solving, students working with an expert model tutor receive feedback as soon as an incorrect formula is entered. In tutorials, students worked solely on their group project with the tutor. Tutors facilitate discussion within small groups and across the whole tutorial group. In this regard, tutors co-produce the project with students, rather than students working on this project in their own time outside of the formal teaching time for the unit. The method, flipped learning, highly encourages a student-centered learning environment, where the student is actively engaged and empowered to take the lead in the learning process. The flipped classroom asks teachers to give up their leading role in teaching in order to encourage students' contributions. It also asks students to change from passive to committed participants and assume the responsibility for their learning.

\section{Fitting}

Where Does Computational Thinking Fit In? OK, so I've talked a bit about the mechanics of teaching computational thinking. But where does computational thinking fit into the standard educational curriculum? The answer, I think, is simple: everywhere! One might think that computational thinking was somehow only relevant to STEM education. But it's not true. Computational thinking is relevant across the whole curriculum. To social studies. To language arts. To music. To art. Even to sports. In every one of these areas there are very powerful-and often very clarifying - things that can be done with computation and computational thinking. And the 
great thing is that it's all accessible to students. The main point is that computational thinking provides a framework that makes things more transparent and easier to understand. When you formulate something computationally, everyone can try it out and explicitly see how it works. There's nothing hidden that the student somehow has to infer from some comment the teacher made. Well, with computational thinking you can imagine creating a social network for the play. And pretty soon you have a nice summary, that's a place to launch from in talking about the nuances of the play and its themes.

\section{Summary}

This paper illustrates an approach which guarantees a high quantity as well as a high quality of learning method as implemented in recent Web 2.0 applications. But thanks to all the technology we've built, one can express things at a much higher level—so one can concentrate on computational thinking, not mere programming. Yes, there's certainly a need for some number of software engineers in the world who can write low-level programs in languages like $\mathrm{C}++$ or Java or JavaScript — and can handle the details of loops and declarations. But that number is tiny compared to the number of people who need to be able to think computationally. In the context of a concrete e-learning platform, a real world scenario has illustrated practical impact of Web 2.0-inspired flipped classroom. Promoting ease of use and cost effectiveness is the theme. Thanks to the modularity and the clear interfaces it should be easily possible to enrich systems that were developed at colleges as well as open-source applications. Pedagogy must be clearly based on theoretical models of learning. Building bridges online is highly desirable. It does strengthen the need for the research outlined here into the effectiveness of flipped classroom to develop a better platform-local, national, and international-for young people. The technology-based instruction should promote learner-centered experiences, which will definitely strengthen students' potential entrepreneurship and innovation.

\section{Acknowledgements}

This research was financially supported by Beijing Information Technology College Research Subject.

\section{References}

[1] Strang Gilber, Linear Algebra and Its Applications[M].Brooks, Cole, 2005, pp55-100.

[2] Anil K. Aggarwal, Web-Based Education: Learning from Experience[M].IRM Press, Hershey, 2003, pp5-10.

[3] Giordani, D. S., Moraes, E. J. C., \& Barreto, Simulation of a competitive business environment: a case study in a Chemical Engineering Program[J]. Production, 2017(27) 